\title{
A Cross-Sectional Study Rating the Perceived Impact and Reach of the Ministry of Health and Wellness Initiatives to Curb Physical Inactivity in Jamaica
}

\author{
Van Der Rowe \\ Faculty of Science and Sport, University of Technology, Jamaica \\ $\square$ Corresponding Author: Van Der Rowe, E-mail: vanderrowe9@gmail.com
}

\begin{tabular}{ll}
\hline ARTICLE INFORMATION ABSTRACT \\
\hline
\end{tabular}

Received: 08 September 2021

Accepted: 01 October 2021

Published: 14 October 2021

DOI: $10.32996 /$ jspes.2021.1.2.2

\section{KEYWORDS}

Physical activity strategies, inactivity, lifestyle, weight loss.
Like other countries, Jamaica in the last 2 decades has seen an increase in physical inactivity, which has significantly increased the number of people living with chronic lifestyle diseases on the island. It can be argued that increased industrialization has resulted in several ecological concerns, such as violence that may deter people from engaging in physical exercise, pollution and polluted air, lack of parks, walkways, and sports/recreational amenities. Despite the causes of physical inactivity, there is a global mobilization by the World Health Organization (WHO) and individual countries to reduce physical inactivity levels by at least $15 \%$ by 2030 . The COVID-19 pandemic has surely put a dent in the physical activity (PA) targets. Many countries initiated restrictive measures (lockdowns, curfews, and social distancing) to try and contain the spread of the COVID-19 virus. For countries such as Jamaica, combating physical inactivity seems to require strategies, interventions, motivation, and the promotion of physical activity (PA) culture from all stakeholders (integrated approach) to meet the WHO's goals and standards by 2030 . The purpose of this research was to assess the perceived impact and reach of Jamaica's Ministry of Health and Wellness (MOHW) initiatives to promote PA, impact weight loss, and lifestyle while trying to reduce sedentary behaviors in Jamaica. A standardized questionnaire was used in a single cross-sectional design that surveyed 843 Jamaicans using Google Forms. Participants ages 12-71 years old were surveyed using a hybrid sample strategy that used convenience and snowballs sampling techniques. The results of the study indicated that there was a $35 \%$ participation rate in the $\mathrm{MOHW}$ initiatives, with only $14.3 \%$ of the participants believed that the initiatives were impacting their weight loss and lifestyle. Additionally, $51.6 \%$ of the participants did not believe the initiatives caused them to participate more in PA; on average, the initiatives by the MOHW were rated at $50 \%$ effective. The initiatives are not very effective in reaching most of the population, which is not achieving the goal of enhancing healthy lifestyle practices and reducing physical inactivity. These implications may potentially see an increase in Jamaica's physical inactivity levels and chronic lifestyle diseases if these initiatives are not intensified to reach the majority of the population.

\section{Introduction}

Physical inactivity raises the risk of several negative health outcomes, including the world's major non-communicable diseases (NCDs) to also include coronary heart disease (CHD), type 2 diabetes, breast, and colon cancers, and shortens life expectancy (Guthold et al., 2018). Furthermore, many countries worldwide seem to be struggling to get sedentary behaviors and physical inactivity under control, which poses a significant public health issue. According to Guthold et al. (2018), inadequate physical exercise is a major risk factor for non-communicable illnesses and has a poor impact on mental health and quality of life. On the other hand, physical activity has several health advantages, including a reduced risk of cardiovascular disease, hypertension,

Copyright: (c) 2021 the Author(s). This article is an open access article distributed under the terms and conditions of the Creative Commons Attribution (CC-BY) 4.0 license (https://creativecommons.org/licenses/by/4.0/). Published by Al-Kindi Centre for Research and Development, London, United Kingdom. 
diabetes, and breast and colon cancer. Furthermore, PA improves mental health, prevents the onset of dementia, and aids in maintaining a healthy weight (Guthold et al., 2018).

In acknowledgment of the close relation between PA and major non-communicable illnesses, the World Health Organization member states committed to a 10\% decrease in the prevalence of inadequate PA by 2025 and $15 \%$ by 2030 as one of nine global objectives to promote non-communicable disease prevention and treatment (Guthold et al., 2018). Monitoring present trends over time of inadequate PA is critical for tracking progress towards the global PA goal and identifying high-risk populations, evaluating policy efficacy, and guiding future policy and program design (Guthold et al., 2018). The 2025 worldwide PA objective (a 10\% reduction in inadequate physical activity) will not be attained if present trends continue. Therefore, prioritizing and scaling up policies to raise population awareness and reducing the level of sedentary behavior is critical (Guthold et al., 2018). The WHO has stepped up its efforts in response to the growing costs and deaths associated with inactivity throughout the world. The WHO responded by launching the Global Action Plan on Physical Activity 2018-2030, promoting PA and track inactivity (Pogrmilovic et al., 2020).

According to WHO (2018), they have been responding to different nations' requests for updated information and a framework of effective and achievable policy initiatives to promote PA at all levels. The WHO strategy outlines four goals and 20 policy recommendations that may be implemented in any country to address the cultural, environmental, and individual factors contributing to inactivity $(\mathrm{WHO}, 2018)$. The four strategic objectives are to create active societies, active environments, active individuals, and active systems (WHO, 2018). While three of the 20 policy recommendations for stakeholders include implementing best practice communication campaigns linked to community-based programs; conducting national and community-based efforts to raise knowledge of the social, economic, and environmental benefits of PA, as well as an appreciation for them; and individuals should have better access to high-quality public and green open spaces, green areas, recreational places (including river and coastal regions), and sports facilities (WHO, 2018).

According to the Department of Health and Human Services (2018), to change the level of physical inactivity in the United States, working one-on-one or in small groups is more effective. Other techniques can be adopted at a community level through programs, practices, and regulations that encourage people to engage in physical exercise. The United States, through their Department of Health and Human Services (2018) and the Centers for Disease Control and Prevention (CDC), has rolled out several strategies aimed at reducing the high level of inactivity within the country. Some of the strategies include guidance from professionals or peers, support from others (friends and family adopting the buddy systems), technology-based approaches (providing virtual coaching to help people set and monitor PA goals), point-of-decision prompts (provide signs or other prompts that encourage people to make an active choice), school policies and practices, access to indoor or outdoor recreation facilities or outlets, and building communities in ways that make it easier for people to be active (Department of Health and Human Services, 2018)

According to WHO (2016), the European Region's PA strategies for 2016-2025 are to promote a life-course approach; empower people and communities through health-enhancing environments and participation; promote integrated, multisectoral, and partnership-based approaches; ensure the adaptability of PA programs (interventions) to different contexts and use evidencebased strategies to promote PA and to monitor ongoing implementation and impact. Research published by Powers et al. (2017) indicated that public health standards, educational initiatives, and structural and budgetary reforms to encourage healthy behaviors have all been used in Australia during the last two decades to lower the high-risk factors that lead to bad lifestyles practices. Powers et al. (2017) also indicated that the prevalence of overweight and obesity, which has been primarily addressed by food industry awareness programs and voluntary actions, has grown significantly across all demographic sub-groups within Australia.

On the other hand, Jamaica continues to struggle with high inactivity rates and deaths from NCDs (Ministry of Health and Wellness Jamaica, 2013). The last Country Card published by the Global Observatory for Physical Activity (GoPA) in January 2021 indicated that Jamaica's PA prevalence was at 67\%, coming from $72 \%$ in 2015 (GoPA, 2021). The recent Country Card published for Jamaica in 2021 saw 246,527 (8.4\%) of Jamaicans dying because of physical inactivity, with NCDs accounting for 79 percent of fatalities (GoPA, 2021). Diabetes and hypertension alone cost Jamaicans US\$460,442,870 in 2001 (direct and indirect costs), accounting for $5.87 \%$ of the country's GDP (Ministry of Health and Wellness Jamaica, 2013). According to a World Bank study in 2013, the overall economic cost on people in Jamaica in 2006 and 2007 was projected to be JM $\$ 47,882$ million (US\$641 million), including indirect income loss (Ministry of Health and Wellness Jamaica, 2013). On the other hand, the first Country Card published in 2015 has intensified the Government of Jamaica and by extension, the Ministry of Health and Wellness (MOHW) initiatives to promote PA and reduce sedentary behaviors. Since then, additional initiatives have been employed, costing the government millions of dollars. The most significant initiative was the launch of a strategic action plan in 2013 by the MOHW to reduce the burden of preventable 
morbidity and disability and avoidable premature mortality due to non-communicable diseases and injuries up to $25 \%$ by 2025 (Ministry of Health and Wellness Jamaica, 2013).

The strategic plan's five priority programs include risk factor reduction and health promotion; comprehensive and integrated disease management for NCDs and injuries; surveillance, research, monitoring, and evaluation; public policy and advocacy and leadership; and governance and capacity building. Some of the most active initiatives currently being employed by the MOHW includes a PA guide toolkit for schools and workplaces; capacity building for health education officers, doctors, nurses, and community personnel; grassroots development in schools (camps and workshops); national school moves day; TV ads promoting $\mathrm{PA}$; the introduction of PA wellness checks for workplaces; TV exercise sessions; and the hiring of four PA specialists for each region (Rowe, 2021). How impactful have the initiatives by the MOHW been in the promotion of PA and the reduction of sedentary behaviors in Jamaica? This research sought to rate the perceived impact and reach of the MOHW initiatives to promote PA, impact weight loss and lifestyle and reduce sedentary behaviors in Jamaica. This study can significantly help the MOHW to evaluate the reach and impact of their implemented initiatives before the COVID-19 pandemic and intensify the most effective strategies for better results.

\section{Methods}

The methods chosen for this research sought to get a unique view of the perception Jamaicans had about the initiatives be employed by MOHW to curb physical inactivity. Furthermore, the study sought a representative sample from all parishes to get a comprehensive understanding of the impact of the initiatives on their PA levels and perceptions of their sedentary lifestyle practices. The purpose of the study was to rate the perceived impact and reach of the MOHW initiatives to curb physical inactivity in Jamaica.

\subsection{Design and Population Sample}

The research utilized a quantitative cross-sectional survey design to collect, analyze and report findings of the study. The target population for this research was Jamaican citizens living in Jamaica before the COVID-19 pandemic. The research surveyed 843 Jamaicans with participants coming from all 14 parishes with ages ranging from 12-71 years. The study used a combined approach to reach participants and collect data, considering the COVID-19 pandemic measures (gathering limits, curfew, and lockdowns) still being enforced by the Government of Jamaica. The combined approach utilized convenience and snowball techniques to reach the research sample size. The convenience technique allowed the researcher to utilize known participants, while the snowball techniques allowed the known participants to refer additional participants for the study.

\subsection{Procedure and Data Collection}

This research followed the preset guidelines by the researcher's university, which requires a proposal to first be submitted to the university's ethics committee for ethical approval. Once ethical approval was granted, the researcher proceeded to create a Google form with structured questions. However, the COVID-19 restrictions being enforced in Jamaica meant that reaching participants would be difficult and the traditional approach would not work. The researcher included a cover page on the Google form that explained the purpose and risk of the study while seeking permission from prospective participants before they were allowed to proceed to the questions in the survey. As it relates to the collection of the data, a strategic combined sampling method was employed to get the best possible chance of reaching the required sample size for the study and ensured that all parishes were represented. The researcher used a hybrid sample strategy that used convenience and snowball sampling techniques to reach the participants. The data was combined on Google and later downloaded to a Microsoft excel sheet, where it was transferred to SPSS for a descriptive analysis to be done.

\subsection{Instrumentation}

The questionnaire for the survey was created in Google forms using a structured approach with 15 multiple-choice items. The survey was administered during a one-week period using snowballing and convenience techniques to reach the sample size. The form included demographic details such as age, gender, parish, and area. The questionnaire also included questions on the initiatives that the participants have participated in, participants' perception of the impact of the initiatives on weight loss and lifestyle, participation in physical activity, participants' perception of the most effective initiative, and participants' overall rating of the MOHW response to the physical inactivity rates in Jamaica.

\subsection{Data Analysis}

The data analysis was done using SPSS version 22 (IBM Statistics) to perform statistical analysis. A Pearson Chi-Square test was used to determine the significance of the relationship between the age of the participants and the impact of the MOHW initiatives on their weight loss and lifestyle. Also, crosstab analysis was done using the variables age, area, initiatives participated in, PA participation before and during the pandemic. The crosstab was also used to generate percentages, tables, and charts necessary for the analysis. 


\subsection{Ethical Consideration}

Ethics is a crucial part of the research process to ensure that the participant's rights, safety, and privacy are always protected. This study adhered to all ethical norms established in research as well as those set out by the University's Ethics Committee. Consent to participate and personal and institutional privacy are common issues that arise during research like this. The researchers took all appropriate precautions to verify that participants provided their permission to engage in this study (the survey needed permission before respondents could advance to the questions) and that no personally identifiable information was asked or acquired from people or institutions. All contributions were kept anonymous, and the researcher made sure the data gathered was encrypted and kept on password-protected devices. Before even being removed or destroyed, the study data will be retained for up to five years.

\section{Results}

The research surveyed 843 persons across all 14 parishes in Jamaica, ranging from ages 12-71 years, to assess the impact of the MOHW initiatives to curb physical inactivity in Jamaica. The results of the study are presented using subheadings from the questionnaire. The Majority of the participants, 629 (74.6\%) were females and 202 (24\%) were males, while another $12(1.4 \%)$ of the participants did not wish to state their gender. As it relates to the parishes, $21.7 \%$ of the participants came from St. Catherine, while $19.5 \%$ of the participants came from St. Andrew. Additionally, $15.3 \%$ and $11.5 \%$ were from Kingston and St. James, respectively. The remaining $32 \%$ of the participants came from the other ten parishes in smaller percentages. Regarding the participants' location in their parishes, the majority of the participants (43.1\%) reside in urban areas, while suburban and rural areas accounted for $28.8 \%$ and $28.1 \%$, respectively.

\subsection{The Ministry of Health and Wellness initiatives to curb inactivity in Jamaica}

3.1.1 The level of participation in MOHW PA initiatives: The MOHW with the aim of curbing physical inactivity in the country, launched its strategic action plan to increase awareness and participation in PA. Over the years, the action plan has implemented several initiatives targeting different areas of society. As a result, the participants were asked to select the most active initiatives. Participants were required to select the activity that they have participated in the most since the intensified efforts by the MOHW in 2013. Jamaica moves initiative received the most indication of participation, with $14.4 \%$ of the respondents indicating that they have participated in that activity. However, 547 (65\%) of the participants have never participated in any of the MOHW initiatives geared towards reducing physical inactivity in Jamaica. The other areas that received some indications of participation were TV exercise workout programs (6.5\%), the national school moves day (4.2\%), PA guide sessions (3.1\%), PA wellness checks (2.1\%), school camps and workshops (2\%), community exercise programs (1.5\%) and capacity building $(0.6 \%)$

3.1.2 The impact of the MOHW initiatives to curb physical inactivity: The respondents were asked if the initiatives by the MOHW to curb inactivity in Jamaica has increased their level of participation in PA. The response from $435(51.6 \%)$ of the respondents indicated that the initiatives by the $\mathrm{MOHW}$ to increase PA participation in Jamaica had not increased their level of participation. This meant that the initiatives are not attractive, they are not targeting all aspects of the population, or it is not giving the population enough reason to participate in PA. On the other hand, 232 (27.5\%) of the respondents indicated that the initiatives by the Government of Jamaica and by extension, the MOHW have positively impacted their participation levels in PA. However, $176(20.9 \%)$ of the respondents indicated that they were undecided as to whether their level of participation was impacted positively by the initiatives of the MOHW or whether their participation level had increased and whether it was because of the MOHW initiatives.

The results showed that the highest indication of no impact on weight loss and lifestyle by the MOHW initiatives was $22.3 \%$ of the respondents in the $32-41$ age group. Another $18.9 \%$ of the respondents came from the $22-26$ age group, $15.8 \%$ from the $42-51$ age group, $14.1 \%$ from the $17-21$ age group, and 10.1\% from the $27-31$ age group, all indicating that the MOHW initiatives did not impact their weight loss or lifestyle.

3.1.3 The age of participants and the MOHW initiatives in which they participated: The majority of the participants (65\%) have not participated in any of the MOHW initiatives to curb physical inactivity in Jamaica. However, for the respondents who participated, they did so in areas such as capacity building with ages ranging from 42-52 years. In addition, participants who participated in community exercise programs were between 32-42 years, while participants who participated in the national school moves day were between 32-52 years. Other areas include PA guide sessions (ages 12-16, 17-21, 32-41, and 52-60 years), PA wellness checks (ages 32-51 years), school camps and workshops (ages 17-21 and 32-41 years), Jamaica moves initiative (ages 2260 years) and TV exercise programs (ages 12-60 years).

3.1.4 The participation levels in urban, suburban, and rural areas: The majority of the respondents (44.2\%) that did not participate in any of the MOHW initiatives to curb physical inactivity in Jamaica either reside in the country's capital (Kingston) or 
in the urban areas of their parish. Another $29.4 \%$ of the participants that did not participate in any PA initiatives were from suburban areas. At the same time, the rural areas accounted for $26.3 \%$ of the participants that did not participate in any of the initiatives. The MOHW initiatives that saw the most participation were the Jamaica Moves initiative (14.4\%) and the TV exercise programs (6.5\%). The Majority of the respondents (45.5\%) that indicated they have participated in Jamaica moves initiative were from urban areas. While Jamaica moves participation in suburban and rural areas accounted for $27.3 \%$ and $27.3 \%$, respectively. The majority of the participants (38.2\%) that indicated they have participated in the TV exercise programs were from urban areas, in comparison to $32.7 \%$ and $29.1 \%$ that came from rural and sub-urban areas, respectively.

\subsection{The Effectiveness of the Ministry of Health and Wellness Initiatives}

The participants were asked to indicate the frequency of their participation in PA before the COVID-19 pandemic; $80 \%$ of the participants indicated that they were frequently participating in PA before the pandemic. While $73.3 \%$ of respondents, when asked about their level of participation in PA during the pandemic, indicated that they were frequently participating. Despite the level of participation in PA before and during the COVID-19 pandemic, 545 (65.3\%) of the participants indicated that the initiatives by the MOHW to curb inactivity in Jamaica did not impact their weight loss or lifestyle. Another $170(20.4 \%)$ of the participants were undecided, which meant they were unsure if the initiatives impacted them or if they had lost weight, or if their lifestyle had changed.

The participants were also asked to indicate the most effective initiative that the MOHW has implemented to curb inactivity in Jamaica; $29.4 \%$ of the respondents were undecided. While $25.4 \%$ of the respondents indicated that Jamaica moves initiative was the most effective. The other areas include TV ads promoting PA (12.2\%), community-based PA (9.4\%), PA wellness checks for workplaces $(7.5 \%)$, PA guide toolkits for schools and workplaces (3.6\%), the national school moves day (2.8\%), grassroots developments in schools (2\%), PA camps and workshops (1.9\%) and capacity building ( $0.6 \%)$. However, $5.2 \%$ of the respondents believed that none of the initiatives by the MOHW were not effective in curbing inactivity in Jamaica.

The participants were also asked to rate the general initiatives by the MOHW to curb inactivity in Jamaica. The majority of the participants (50.3\%) gave the government initiatives a score between 0-49\%. At the same time, the other participants (49.7\%) gave the government a score between $50-100 \%$. The average score given rated the initiatives at $50 \%$. Many of the respondents who gave the government initiatives a failing grade has never participated in any of the initiatives by the government. The majority of the participants (65\%) that has never participated in any of the MOHW initiatives gave the government a score between $0-50 \%$; this was represented by 326 of the 547 participants that had never participated in any of the MOHW initiatives to curb physical inactivity in Jamaica. The other $35 \%$ of the 547 participants that have never participated in any of the initiatives still gave the MOHW initiatives a passing grade between $50-100 \%$

\section{Discussion}

This section aims to explain, clarify, and compare the study's results to those of other studies using the survey's research questions. Additionally, the discussions sought to examine other interventions and strategies that can be pursued by the MOHW to help curb physical inactivity in Jamaica. The study's goal was to assess the perceived impact and reach of the Ministry of Health and Wellness' programs to combat physical inactivity in Jamaica. The discussion for this section is guided by the research questions of the study.

\subsection{Has the Ministry of Health and Wellness initiatives to curb inactivity in Jamaica increased the level of participation in Physical Activity?}

The results of this study indicated that there was an $80 \%$ participation level in PA by Jamaicans before the pandemic. However, the level of participation in PA during the pandemic was $73.3 \%$. Gjaka et al. (2021) study also found similar results in PA participation when compared to the pre-home-confinement conditions. Gjaka et al. (2021) further explained that the COVID-19 home confinement had a negative effect on PA levels during their study. This resulted in a significant decrease in days, minutes/day, and energy expenditure for three types of PA (vigorous intensity, moderate intensity, and walking activity) and the overall PA level expressed as energy expenditure (Gjaka et al., 2021).

Despite the level of participation before and during the COVID-19 pandemic, most participants (65\%) in this study indicated that they have never participated in any of the MOHW initiatives to curb inactivity in Jamaica. However, the other $35 \%$ of the participants indicated that they have participated in at least one initiative; the highest percentage responses in participation were seen in Jamaica moves initiative (14.4\%), TV exercise programs (6.5\%), and the national school moves day (4.2\%). Research published by Tuso (2015) has corroborated the results of this study with findings indicating that no one intervention would boost PA rates. Instead, a set of interventions acting in harmony may be the catalyst needed to get people moving. Tuso (2015) further explained that encouraging people to be physically active for at least 150 minutes per week; establishing healthy settings by making it simpler for people to be physically active where they live, learn, work, play, and worship; assessing PA as a vital sign; monitoring the illness incidence of people who are physically active verse those who are not, and disseminate best practices are all strategies that can be used to curb physical inactivity. 
The results of this study also indicated that the majority of the respondents (51.6\%) did not think the initiatives by the MOHW to increase PA activity participation in Jamaica have increased their level of participation in PA. Some of the respondents were undecided about whether the initiatives impacted them or if their participation levels had changed. The participants that indicated no weight loss or lifestyle changes because of the MOHW initiatives were represented by $22.3 \%$ of the respondents in the $32-41$ age group. Jamaica moves initiative and the TV exercise programs accounted for participation across all age groups in all parishes. Jorstad and Piek's (2021) study indicated that when the country is unlocked, health care practitioners and scientific policymakers, professional associations, and government agencies should prioritize to obtain better levels of sports and exercise than before the country's lockdown. Jorstad and Piek's (2021) research further explained that public campaigns, TV ads, and other forms of promotional initiatives are needed to reduce the level of inactivity within the country. Lack of exercise and physical inactivity continues to be major issues in our culture, resulting in a curve of inactivity that we must flatten collectively and over time (Jorstad and Piek, 2021).

\subsection{How effective are the Ministry of Health and Wellness initiatives to curb inactivity in Jamaica?}

Despite the levels of PA engagement before and during the COVID-19 pandemic, 545 (65.3\%) of the participants believed the MOHW's efforts to reduce inactivity in Jamaica did not affect their weight loss or lifestyle. Another 170 (20.4\%) of the participants were indecisive, indicating that they were unclear whether the initiatives influenced them if they had lost weight, or whether their lifestyle had changed. Sandoval et al. (2021) believe that lifestyle changes do not have to be drastic; obesity and diabetes trends may stabilize if the population adopts a lifestyle consistent with government diet guidelines. Sandoval et al. (2021) further explained that a mixed approach that incorporates intermittent fasting, nutritional supplements consumption, and resistance exercise might be good strategies for the government to implement in helping to control weight loss and lifestyle. The results of this study also indicated that most of the participation in the MOHW initiatives was mostly centered in the urban areas. Additionally, the two activities that saw the most participation were the Jamaica moves initiative and the TV exercise programs. The results also indicated that the most effective of the two based on participants' responses is the Jamaica moves initiative. On average, the MOHW initiatives to curb inactivity in Jamaica were rated at $50 \%$ effective.

\section{Conclusion}

The aim of the study was to assess the perceived impact and reach of the MOHW initiatives to promote PA, impact weight loss and lifestyle and reduce sedentary behaviors in Jamaica. The initiatives by the MOHW have seen a 35\% participation rate, with many of these participants engaging in the Jamaica moves initiative and the TV exercise programs. Despite the low participation in the MHOW initiatives to curb inactivity in Jamaica, the level of PA participation before the pandemic was $80 \%$, while during the pandemic, it was at $73.3 \%$. However, $51.6 \%$ of the participants did not believe that the initiatives by the MHOW to curb inactivity in Jamaica were helping to increase their level of participation in PA. In comparison, $20.9 \%$ of the respondents were undecided as to whether the initiative influenced their level of participation.

Additionally, only $14.3 \%$ of the participants believed that MOHW initiatives impacted their weight loss and lifestyle. Furthermore, some of the initiatives seemed to have reached all the age groups. However, there is a very low percentage of participation and a low impact on the different age groups. Additionally, the majority of participants that did not participate in any of the initiatives by the government reside in urban areas. The results also showed that the most effective initiative by the MOHW were the Jamaica moves and the TV exercise programs. On average, the initiatives by the MOHW to curb inactivity in Jamaica were rated at $50 \%$ effective. A positive point to note from this study is the level of awareness shown by participants for the MOHW initiatives, however, the type of encouragement and motivation to participate is missing.

Despite the results of this study, there were some limitations. The study did not use the traditional way of collecting data which may have influenced the results. Only persons who had internet or were able to maneuver the survey platform participated in the study. The research was done during the pandemic and required participants to recall what was previously being done regarding PA. The recollection and the current initiatives may have influenced how participants responded to the questions. For future studies, an investigation of Jamaica's PA rates after the pandemic ends could give a better understanding of the impact of the pandemic on PA levels in Jamaica.

\section{References}


[1] Department of Health and Human Services (2018). Physical activity guidelines for Americans. centers for disease control and prevention (CDC). Retrieved from https://health.gov/sites/ default/files/201909/Physical_Activity_Guidelines_2nd_edition.pdf\#page =94

[2] Gjaka, M., Feka, K., Bianco, A., Tishukaj, F., Giustino, V., Parroco, M. A., Palma, A., \& Battaglia, G. (2021). The effect of covid-19 lockdown measures on physical activity levels and sedentary behavior in a relatively young population living in Kosovo. Journal of Clinical Medicine, 10, 1-15. https://doi.org/10.3390/jcm10040763.

[3] GoPA. (2021). Global Observatory for physical activity. Retrieved from https://www.ispah.org/councils/global-observatory-for-physicalactivity/

[4] Guthold, R., Stevens, A. G., Riley, M. L., \& Bull, C. F. (2018). Worldwide trends in insufficient physical activity from 2001 to $2016:$ a pooled analysis of 358 population-based surveys with 1.9 million participants. Lancet Glob Health, 6, e1077-1086. DOl:https://doi.org/10.1016/S2214-109X(18)30357-7

[5] Jorstad, H.T., \& Piek, J. J (2021). Physical inactivity in times of a pandemic: another curve to flatten. Neth Heart J, $29,241-242$. https://doi.org/10.1007/s12471-021-01576-5.

[6] Ministry of Health (2013). National strategy and action plan to prevent and control non-communicable diseases (NCDs) in Jamaica 2013 2018. Retrieved from https://www.moh.gov.jm/wp-content/uploads/2015/05/National-Strategic-and-Action-Plan-for-the-Prevention-andControl-Non-Communicable-Diseases-NCDS-in-Jamaica-2013-2018.pdf

[7] Pogrmilovic, K. B., Varela, R. A., Pratt, M., Milton, K., Bauman, A., Biddle, S. J. H., \& Pedisic, Z. (2020). National physical activity and sedentary behavior policies in 76 countries: Availability, comprehensiveness, implementation, and effectiveness. International Journal of Behavioral Nutrition and Physical Activity, 17, 1-13. doi:http://dx.doi.org/10.1186/s12966-020-01022-6

[8] Powers, R. J., Loxton, D., Anderson, E. A., Dobson, J. A., Mishra, D. G., Hockey, R., \& Brown, J. W. (2017). Corrigendum to: Changes in smoking, drinking, overweight and physical inactivity in young Australian women 1996-2013. Health Promotion Journal of Australia, 28, 255-259. http://dx.doi.org/10.1071/HE16085

[9] Rowe, V. (2021). An Investigation into the Development of a National Physical Activity Policy for Jamaica: Physically Fit Jamaica. Journal of Physical Activity Research, 6(1), 21-27. DOI:10.12691/jpar-6-1-4

[10] Sandoval, C., Villagrán, F., Santibañez, S., \& Vargas, C. (2021). Intermittent fasting, nutritional supplementation, and resistance training as a strategy for bodyweight loss: An update. Int. J. Morphol., 39(1), 172-178.

[11] Tuso, P. (2015). Strategies to increase physical activity. The Permanente Journal, 19(4), 84-88. DOI: 10.7812/TPP/14-242.

[12] WHO (2016). Physical activity strategy for the WHO European region 2016-2025. World health organization regional office for Europe. Retrieved from https://www.euro.who.int/_data/assets/pdf_file/0014/311360/Physical-activity-strategy-2016-2025.pdf

[13] WHO (2018). More active people for a healthier world. The global action plan on physical activity $2018-2030$. Retrieved from https://www.who.int/news-room/initiatives/gappa

[14] WHO (2020). Physical activity. Retrieved from https://www.who.int/news-room/fact-sheets/detail/physical-activity 